\title{
Zur Frage qualitativer Änderungen von Lipoproteiden bei der experimentellen Hyperlipämie
}

\author{
Von F. Scheiffarth, H. Götz und H. Griehl \\ Aus der. Abteilung für klinische Immunologie des Universitätskrankenbauses Erlangen-Nürnberg \\ (Vorstand: Prof. Dr. F. Scheiffartb)
}

(Eingegangen am 26. Mai 1966)

\begin{abstract}
Unter dem Einfluß einer tierexperimentell exzeugten Hyperlipämie konnte festgestellt werden, daß die immunoelektrophoretisch differenzierbaren Lipoproteide des Kaninchenserums unter der zunehmenden Lipämie sowohl quantitative als auch qualitative Änderungen aufweisen. Die papierelektrophoretisch faßbaren Besonderheiten liegen lediglich in der quantitativen Zunahme der mit Sudanschwarz färbbaren $\beta$-Lipoproteidfraktion. Hier zeigen sich quantitative bzw. zeitlich unterschiedliche Verhaltensweisen zwischen den intravenös und subkutan behandelten Tieren. Die Befunde werden unter Berücksichtigung des einschlägigen Schrifttums diskutiert.

An increase in lipaemia, due to experimental hyperlipaemia in rabbits, is accompanied by quantitative and qualitative changes in the serum lipoproteins, which can be differentiated by immunoelectrophoresis. In paper electrophoresis, the only detectable change is an increased level of the $\beta$-lipoprotein that stains with Sudan Black. In this respect there are also quantitative and temporal differences between animals treated intravenously or subcutaneously. The findings are discussed with reference to the relevant literature.
\end{abstract}

Studien über die experimentelle Hyperlipämie, wie sie in der einschlägigen Literatur (siehe bei 1) mitgeteilt wurden, führten zu der Erkenntnis, daß unter der Einwirkung eines Netzmittels eine allgemeine Änderung der Zellgrenzflächen im Sinne einer Permeabilitätssteigerung mit Freisetzung zellständiger Lipo- und Glykoproteide und deren Einschwemmung in die Blutbahn erfolgt. Im Serum läßt sich dabei zunächst ein Anstieg der Neutralfette und zu einem späteren Zeitpunkt auch des Cholesterins und der Phosphatide, papierelektrophoretisch ein Anstieg vornehmlich $\operatorname{der} \beta$-Lipoproteide nachweisen. Das gilt nicht nur für die Versuche mit einmaliger Applikation eines Netzmittels, sondern auch für diejenigen mit einer Langzeitbehandlung und einer damit erzeugten Dauerlipämie $(1,2)$. Die im Serum nachweisbare Fettanreicherung wird im wesentlichen als Transporthyperlipämie $(3,4)$ aufgefaßt. Bisherige immunologische Studien über Serumlipide in Patientenseren sowie nach Fettinfusionen (5) haben einige Besonderheiten im quantitativen und auch qualitativen Verhalten der darstellbaren Lipoproteide aufgezeigt.

In der vorliegenden Arbeit sollte deshalb die Frage nach etwa gesetzmäßigen quantitativen und insbesondere qualitativen Äußerungen der lipidtragenden Serumproteide unter dem Einfluß eines unphysiologischen Fettangebotes angegangen werden. Gleichzeitig sollte geprüft werden, ob der Applikationsmodus bzw. die zeitliche Entwicklung der Hyperlipämie die Bindụngsund Transportkapazität der entsprechenden Serumproteine entscheidend mitbeeinflussen.

\section{Methodik}

Versuchsanordnung

Es wurden 4 Kaninchen in Versuch genommen. 2 bekamen pro kg Körpergewicht $2,5 \mathrm{ml}$ einer 50 proz. Lösung von Triton ${ }^{1}$ ) in 0,9 proz. $\mathrm{NaCl}-\mathrm{L}$ ösung intravenös, die beiden anderen $5,0 \mathrm{~m} / \mathrm{kg}$ subkutan als einmalige Injektion appliziert.

Vor Versuchsbeginn sowie nach 2 Stdn., danach in Abständen von 4 Stdn. bis zu 24 Stdn., von da an in jeweils 8stdg. Abständen bis zum 10. Tag weiterhin alle 24 Stdn. bis zum 26. Tag nach Versuchs-

i) Polymeres p-Isooktylpolyoxyäthylen der Fa. Bayer, Leverkusen. beginn wurde den Tieren jedesmal 2,0 m/ Blut entnommen. Nach 2stdg. Stehen wurde das Blut bei 1200 U./Min. 20 Min. lang zentrifugiert. Das gewonnene Serum untersuchten wir mit folgenden Methoden: Papierelektrophorese und Immunelektrophorese, jeweils mit Protein- und Lipoidfärbung. Die Immunelektrophorese wurde vor allem zur qualitativen Differenzierung der Lipoproteide gewählt.

Chemische Blutfettbestimmungen wurden unter Berücksichtigung der relativ häufigen Blutentnahmen nicht vorgenommen; zudem sind die Blutfettrelationen, wie oben erwähnt, bereits mehrfach studiert worden.

Bei jeder Blutentnahme wurden schließlich periphere Blutbilder angefertigt, da sich in früheren Versuchsreihen Auffälligkeiten im Blutstatus gezeigt hatten.

Untersuchungsmethoden

Immunelektrophorese nach der gering modifizierten Vorschrift von Grabar und Williams $(6,7,8)$ mit kombinierter Lipoid- und Proteinfärbung $(9,10)$. Zur Anwendung kamen dabei ein AntiKaninchenserum von der $Z_{\text {iege }}{ }^{2}$ ) sowie versuchsweise AntiHuman- $\alpha$-Lipoproteid- und $-\beta$-Lipoproteidseren ${ }^{2}$ ).

Papierelektrophorese nach den Angaben von Grassmann und HanNig (11) mit Protein- und Lipoidfärbung $(10,12)$.

Das periphere Blutbild wurde in üblicher Weise angefertigt.

\section{Ergebnisse}

Immunelektrophorese

\section{Lipoproteide}

In einem normalen Kaninchenserum kommen unter Verwendung. eines spezifischen polyvalenten Anti-Kaninchenserums und nachfolgender Färbung mit Sudanschwartz B zwei Lipoproteidfraktionen zur Darstellung: ein $\alpha_{1}$ - sowie ein $\alpha_{2}$-Lipoproteid (Abb. 1). Eine Differenzierung von $\alpha_{1}$-Lipoproteiden gelingt nicht. Unter dem Einfluß der Lipämie lassen sich folgende Besonderheiten an den Lipoproteidfraktionen feststellen: Es kommt zur Darstellung eines intensiv gefärbten Präzipitatbogens, der sich von der Auftragestelle bis in den Bereich der $\alpha_{2}-$ Globuline erstreckt und eine Doppelung aufweist (Abb. 2). Außerdem findet sich unmittelbar um und vor der Auftragestelle ein Lipoidfleck ohne Präzipitatbilldung. Mit zunehmender Lipämie verstärken sich das Präzipitat sowie der beschriebene Fleck. Außerdem ver-

2) Behringwerke, Marburg/Lahn. 


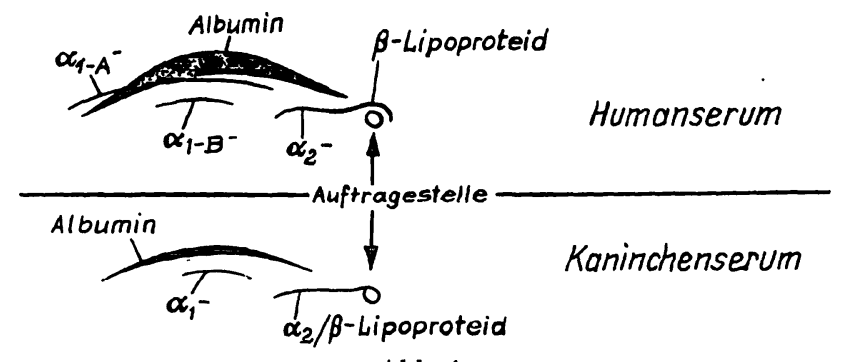

Abb. 1

Halbschematische Darstellung der immunoelektrophoretisch demonstrierbaren Lipoproteide des Serums vom Menschen (oben) und des Kaninchen-Serums (unten)

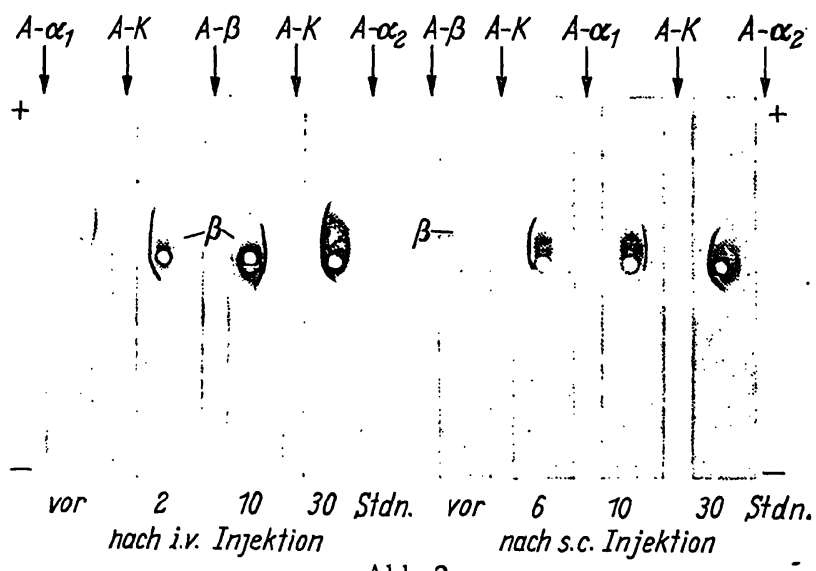

Abb. 2

Verhalten der Lipoproteidfraktionen unter zunehmender Fetteinschwemmung in das Serum verschieden behandelter Kaninchen: links intravenöse Applikation eines Netzmittels, techts subkutane Applikation desselben Netzmittels. Immunelektrophoretische Analysen unter Verwendung eines polyvalenten Anti-Kaninchenserums sowie monovalenter Antiseren gegen verschiedene Lipoproteidfraktionen des Menschen. Färbung mit Sudanschwarz B A- $\alpha_{1}=$ Anti- $\alpha_{2}-$ Lipoproteidserum gegen Mensch
A- $\alpha_{2}=$ Anti- $\alpha_{2}$-Lipoproteidserum gegen Mensch
A- $\beta=$ Anti- $\beta$-Lipoproteidserum gegen Mensch
A-K $=$ polyvalentes Anti-Kaninchenserum

liert die gedoppelte Präzipitationslinie an Wanderungsfähigkeit. Die im Serum unbehandelter Kaninchen nachweisbare Präzipitationslinie im $\alpha_{1}$-Bereich ist nur noch im 2-Stdn.-Wert der Tiere, die Triton i. v. erhielten, als Spur nachweisbar. Sie wird in sämtlichen weiteren Proben völlig vermißt.

Unter Berücksichtigung des unterschiedlichen Applikationsmodus von Triton lassen sich auf Grund der vorliegenden Ergebnisse lediglich quantitative, d. h. Intensitäts-Unterschiede nachweisen, nicht aber qualitative.

Erwähnenswert erscheint die Beobachtung, daß mit monovalentem Anti- $\beta$-Lipoproteidserum gegen Humanproteine im Kaninchenserum adäquate Präzipitate nachweisbar werden.

\section{Proteine}

Die kombinierte Lipoproteid-Protein-Färbung gestattet aufgrund der unterschiedlichen Farbnuancen auch bei Verwendung von Sudanschwarz B und Amidoschwarz 10B eine sichere Zuordnung der Lipoproteidsysteme innerhalb des Präzipitatmusters der immunoelektrophoretisch analysierten Seren. Man erkennt hier im ubrigen auch die mit Antihuman- $\beta$-Lipoproteidserum schwach dargestellte gedoppelte Präzipitationslinie.

\section{Papierelektrophorese}

\section{Lipoproteide}

Die Lipoproteide unbehandelter Kaninchen lassen sich nach papierelektrophoretischer Trennung und nachfolgender Färbung mit Sudanschwarz B in ein Albumin/ $\alpha_{1}$-Globulin-, eine $\alpha_{2}$-, eine $\beta$-Globulin-Fraktion sowie in einen Komplex, der als „Schleppe" von den $\beta$-Globulinen bis zum Auftragestrich reicht, differenzieren. Sämtliche Komponenten sind nur schwach anfärbbar. Nach Injektion von Triton erfolgt insgesamt ein Anstieg der Serumlipide, insbesondere aber $\operatorname{der} \beta$-Fraktion. In der Gruppe der intravenös behandelten Kaninchen ist bereits nach 2 Stdn. eine deutliche Zunahme der $\beta$ Lipoproteide wahrnehmbar. Bis zu $78 \mathrm{Stdn}$. nimmt die Konzentration dieser $\beta$-Lipoproteide kontinuierlich $z u$. Im weiteren Verlauf wird ein Rückgang der Lipidkonzentration festgestellt, wobei sich die Konzentration $\operatorname{der} \beta$-Fraktion $208 \mathrm{Stdn}$. nach Applikation von Triton weitgehend den Normalwerten genähert hat.

Bei den Tieren, welche Triton in doppelter Quantität subkutan injiziert bekamen, war ein Anstieg der Lipoproteide erstmalig nach 6 bzw. 10 Stdn. nachweisbar. Von diesem Zeitpunkt an war eine kontinuierliche $\mathrm{Zu}$ nahme bis zu $104 \mathrm{Stdn}$. nach Applikation zu verzeichnen. Ein Absinken der $\beta$-Lipoproteidwerte konnte in dem weiteren Beobachtungszeitraum nicht sicher festgestellt werden. Selbst bei nach 26 Tagen entnommenen Blutproben waren die Seren noch deutlich lipämisch (Abb. 3).

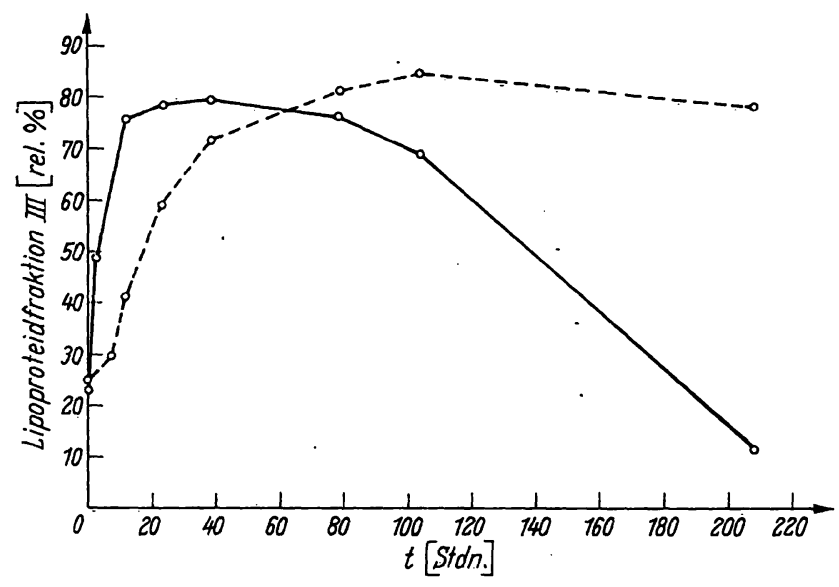

Abb. 3

Graphische Darstellung des zeitlichen Verlaufs der Konzentration der papierelektrophoretisch faßbaren Lipoidfraktion III ( $\beta$ Fraktion) in den beiden, unterschiedlich mit einem Netzmittel behandelten Tiergruppen.

- - - o Gruppe der subkutan behandelten Kaninchen 0 Gruppe der intravenös behandelten Kaninchen

\section{Proteine}

Nach Färbung mit Amidoschwarz 10B und Auswertung der gewonnenen Diagramme konnten bei sämtlichen Versuchstieren keine nennenswerten Verschiebungen in der Serumprotein-Relation festgestellt werden. Lediglich die $\beta$-Globuline zeigten eine mäßige, kontinuierliche Zunahme, die jedoch nicht signifikant war. 


\section{Peripheres Blutbild}

Das periphere Blutbild unbehandelter Kaninchen ist bekanntlich durch eine verhältnismäßig große Streubreite in der Erythrocytenzahl (4-6 Mill./ $\mathrm{mm}^{3}$ ) sowie durch das Vorherrschen kleiner Lymphocyten im weißen Differentialblutbild ausgezeichnet. Während der Versuche traten in der Zahl der Erythrocyten keine Abweichungen auf, dagegen konnte im Vergleich zu den Ausgangswerten für die Leukocyten $\left(5-8000 / \mathrm{mm}^{3}\right)$ eine Leukocytose mit Werten um $13000 / \mathrm{mm}^{3}$ festgestellt werden. Im Differentialausstrich fiel darüber hinaus eine Eosinophilie auf. Ferner fanden sich mit Einsetżen der lipämischen Trübuñg im Tierserum Hämolyse, Anisocytose, Poikilocytose sowie Hypochromasie der Erythrocyten, gelegentlich auch „target cells“.

\section{Diskussion}

Die zur Aufklärung qualitativer Änderungen der Serumlipoproteide bei experimenteller Hyperlipämie vorgenommenen immunoelektrophoretischen Studien haben eine Reihe beachtenswerter Befunde ergeben.

Zunächst sei in Kürze auf die normalen Verhältnisse der Serumlipoproteide des Kaninchens eingegangen: Die Serumlipoproteide unbehandelter Tiere lassen sich immunoelektrophoretisch unter Verwendung eines polyvalenten Anti-Kaninchenserums in eine schwach färbbare Fraktion des Albumin/ $\alpha_{1}$-Globulin-Bereiches sowie in eine langgezogene Präzipitationslinie differenzieren, die sich vom Auftragestrich bis in den $\alpha_{2}$-Bereich erstreckt. Vergleicht man die Lipoproteide des Kaninchenserums mit den Serumlipoproteiden des Menschen, so finden sich eindeutige Parallelen; die Serumlipide des Kaninchens scheinen jedoch in geringerer Konzentration vorzuliegen. Auch gelingt es nicht, die $\alpha_{1}$-Lipoproteide in eine $\alpha_{1-A}$ und in eine $\alpha_{1-B}$-Fraktion zu unterteilen.

Unter der zunehmenden Einschwemmung von Lipiden in das Serum konnte eine intensivere SudanschwarzFärbung der $\alpha_{2} / \beta_{1}$-Lipoproteidlinie festgestellt werden. Außerdem fand sich eine Doppelung und insgesamt eine Verlangsamung der elektrophoretischen Mobilität dieses Präzipitats. Nach den Erfahrungen der Humanpathologie (5) entspricht dieses Phänomen einer Vermehrung von Cholesterin sowie Phospholipiden $(1,3,5)$. Die Verlangsamung der elektrophoretischen Beweglichkeit dieses Lipoidkomplexes ist im wesentlichen mit der quantitativen Anreicherung dieser Ljpoproteide zu erklären, womit zugleich der Makroglobulincharakter der $\alpha / \beta$-Lipoproteide (13) unterstrichen wird.

Die weitere Beobachtung, daß die im Albumin $/ \alpha_{1}$-Globulin-Bereich nachweisbare Lipoproteidfraktion unter zunehmender Fetteinschwemmung abnimmt und schließlich vermißt wird, findet ebenfalls eine Parallele zur Humanpathologie: Bei Fett-Transportstörungen bzw. auf der Höhe einer alimentären Lipämie oder einer Fettinfusion ist die $\alpha_{1}$-Lipoidfraktion - hier insbesondere die menschliche $\alpha_{1-\mathrm{B}}$-Fraktion - vermindert oder überhaupt nicht mehr nachweisbar. Es werden Zusammenhänge dieser Fraktion mit dem Chylomikronentransport diskutiert $(5,14)$.

Aufgrund der Beziehungen des Lipidstoffwechsels zu den Fett-Transportglobulinen findet ein reger Lipidaustausch zwischen den einzelnen Lipoproteidfraktionen statt (15), wobei nach Fettzufuhr im wesentlichen ein Abbau von Neutralfetten und eine Anreicherung von Cholesterin und Phosphatiden erfolgt. Da aber die Neutralfette, die im übrigen den Glyceriden der Chylomikronen entsprechen (14), an die $\alpha_{1}$-Lipoproteide (5, 14), zum Teil auch an $\alpha_{2}$-Globuline (5) gebunden sind, nimmt die Konzentration dieser Lipoproteide mit dem Abbau der Neutralfette sukzessive ab. Die Anreicherung der frei gewordenen Phosphatide und des Cholesterins führt nunmehr zu einer Anlagerung von $\beta$-Globulinen (16); dies findet in der auffallend starken Darstellung und Sudanfärbbarkeit des Präzipitats $\operatorname{der} \alpha_{2} / \beta$-Lipoproteide seinen Ausdruck.

Die Tatsache, daß auch bei Verwendung eines speziesfremden, hier des Anti-Human- $\beta$-Lipoproteidserums eine positive Präzipitationsreaktion auftrat, spricht für eine immunologische Verwandtschaft der Lipoproteide des Kaninchenserums mit denjenigen des Menschen. Solche Verwandtschaftsreaktionen unter speziesfremden Serumproteinen sind insbesondere für Albumin bekannt. Sie wurden bisher nur vorwiegend mit polyvalenten Antisera aufgezeigt, unseres Wissens aber nicht mit Antisera gegen isolierte Subfraktionen.

Die papierelektrophoretischen Befunde stimmen weitgehend mit früheren eigenen Untersuchungsergebnissen $(1,3,4)$ überein. In vorliegender Versuchsanordnung konnte für die $\beta$-Lipoproteidfraktion darüber hinaus der unterschiedliche Effekt bei intravenöser und subkutaner Applikation des Netzmittels demonstriert werden.

Die Beobachtungen am peripheren Blutbild stehen hinsichtlich der Leukocytose ebenfalls im Einklang mit früheren entsprechenden Ergebnissen (1). Der beobachteten Hämolyse scheint insofern eine Bedeutung zuzukommen als sie wahrscheinlich Ausdruck der allgemeinen Permeabilitätsstörung unter dem verabfolgten Netzmittel ist.

\section{Literatur}

1. Scheiffarth, F., H. Götz, H. Schön, G. Berg und W. SCHULZE, diese Z. 2, 150 (1964). - 2. MEYER, W. W., Klin. Wschr. 30, 245 (1952). - 3. Schön, H. und G. Berg, a) Arzneimittelforsch. Aulendorf 7, 307 (1957); b) Blut 5, 297 (1959). - 4. SchöN, H., G. BERG und M. GMäHLICH, Arzneimittelforsch. Aulendorf 8, 768 (1958). - 5. BerG, G., a) Verh. Dtsch. Ges. Inn. Med: 70, 971 (1964); b) Åtztl. Laborat. 11, 298 (1965). — 6. Göтz, H., G. Berg und F. ScheIfFarth, Z. Immunit.forsch. 114, 72 (1957). - 7. Grabar, P., Bull. Soc. Chim. Biol. 36, 65 (1954). -
8. Grabar, P. und C. A. Williams, Biochem. biophysica Acta (Amsterdam) 10, 193 (1953). - 9. Grabar, P. und P. Burtin, a) Analyse Immuno-électrophorétique. Masson u. Cie., Paris (1960); b) Immunoelektrophoretische Analyse. Elsevier Publ. Comp., Amsterdam-London-New York (1964). - 10. Henning, N., Klinische Laboratoriumsdiagnostik, 3. Aufl., Urban u. Schwarzenberg, München-Berlin (1966). - 11. GrassmanN, W., K. HanNig und M. Knedex, Dtsch. Med. Wschr. 76, 333 (1951). - 12. Scherffarth, F., G. Berg und H. Götz, Papierelektrophorese in 
Klinik und Praxis. Urban u. Schwarzenberg, München-Berlin (1962). - 13. KORNGOLD, L. und R. LIPARI, Science (Washington) 121, 170 (1955). - 14. Hitzig, W. H., Die Plasmaproteine in der klinischen Medizin. Springer, Berlin-Göttingen-Heidelberg (1963).
- 15. Lindgren, F. T. und A. V. Nrchols, in: The Plasma Proteins, Vol. 2, S. 2, Hrsg. F. W. Purnam, Academic Press New York u. London (1960). - 16. NrkkIL̈̈, E., Scand. J. Clin. Lab. Invest. 5, 1 (1953).
Prof. Dr. F. Scheiffarth

Abteilung für klinische Immunologie des Universitätskrankenhauses 852 Erlangen, Krankenhausstr. 12

\title{
Isoenzyme von Lactat-Dehydrogenase im Sternalmark- und Venenblutserum bei inneren Krankheiten ${ }^{1}$ )
}

\author{
Von Z. Churý, J. Továrex und J. VojtKová \\ Aus dem Institut für Pathologische Pbysiologie (Direktor: Prof. Dr. J. Vasku) und der 3. Medizinischen Klinik (Direktor: \\ Prof. Dr. Dr. J. Pojer) der Purkyne-Universität Brno, Tschechoslowakei
}

(Eingegangen am 11. Mai 1966)

Es wird über vergleichende Untersuchungen von Lactat-Dehydrogenase und deren Isoenzymen im Serum des Knochenmarks und des venösen Blutes bei 44 hämatologischen und internistischen Patienten berichtet. Bei 10 Patienten wurden auch die Isoenzyme in den Erythrocyten und kernhaltigen Zellen aus dem Knochenmark untersucht. In Seren des Knochenmarks wurden höhere Aktivitäten der LDH und deren Isoenzyme, besonders von $\mathrm{LDH}_{3}$ bis $\mathrm{LDH}_{5}$ gefunden. Diese Konzentrationszunahme war dem Grad der Hämolyse nicht streng proportional. Bei einigen Patienten, bei welchen die Knochenmarksseren ganz klar und nicht hämolytisch waren, waren die Aktivitäten von $\mathrm{LDH}$ und auch $\mathrm{LDH}_{3}$ bis $\mathrm{LDH}_{5}$ größer als in venösen Seren desselben Patienten. Dịe Autoren sind deshalb der Meinung, daß diese Aktivitätszunahme nicht nur aus den hämolysierten Erythrocyten, sondern auch aus den kernhaltigen Knochenmarkszellen stammte.

Comparative studies are reported on lactate dehydrogenase and its isoenzymes in the serum of bone marrow and venous blood from 44 haematological and internal patients. The isoenzymes in the erythrocytes and nucleated cells from the bone marrow from 10 patients were also studied. The serum of bone marrow contained higher activities of $\mathrm{LDH}$ and its isoenzymes, especially $\mathrm{LDH}_{3}, \mathrm{LDH}_{4}$ and $\mathrm{LDH}_{5}$. There was no strict proportionality between the increase in concentration and the degree of hemolysis. In some cases, where the bone marrow serum was clear and non-hemolytic, the activities of $\mathrm{LDH}, \mathrm{LDH}_{3}, \mathrm{LDH}_{4}$ and $\mathrm{LDH}_{5}$ were. higher than in the venous serum of the same patients. We therefore believe that this increase in activity not only arises from the hemolysed exythrocytes, but also from the nucleated bone marrow cells.

Bei dem heutigen Aufschwung der klinischen Enzymologie ist es erstaunlich, wie wenig Aufmerksamkeit man der Untersuchung von Enzymen im Serum des Knochenmarks widmet, wenn auch einige Ergebnisse bezeugen, daß wenigstens ein Teil der Serumenzyme bei einigen Erkrankungen (1) aus dem KM in das periphere Blut ausgeschwemmt werden dürfte. HeLler und Mitarb. (2) berichteten über die Erhöhung von einigen Enzymen einschließlich Lactat-Dehydrogenase ${ }^{2,3}$ ) im Plasma des KM von 4 Patienten mit posthämorthagischer Anämie und noch höhere Aktivitäten bei einem $\mathrm{Pa}$ tienten mit megaloblastischer Anämie. Auch wir (3) beobachteten bei 20 Patienten (15 mit hämatologischen und 5 mit inneren Erkrankungen) eine über die Norm des venösen Serums erhöhte Aktivität von LDH im Serum des KM. Wir vermuteten deshalb, daß minde-

1) Teilergebnisse wurden vorgetragen auf der Arbeitsversammlung der Hämatologischen Gesellschaft vom 12.-13. November 1965 in Ostrava (5).

2) Der Trivialname Lactat-Dehydrogenase wird hier gebraucht für das Enzym L-Lactat: NAD Oxydoreduktase, EC 1.1.1.27, Glutamat-Pyruvat-Transaminase für L-Alanin: $\alpha$-Oxoglutarat Aminotransferase, EC 2.6.1.2, Glutamat-Oxalacetat-Transaminase für L-Aspartat: $\alpha$-Oxoglutarat Aminotransferase, EC 2.6.1.1, Aldolase für: Fructose-1,6-Diphosphat D-Glyceraldehyd-3-Phosphatlyase, EC 4.1.2.7, Phosphohexoisomerase für D-Gluçose-6-phosphat $\mathrm{Ke}$ tol-Isomerase, EC 5.3.1.9, Malatdehydrogenase für L-Malat: NAD Oxydoreduktase, EC 1.1.1.37.

3) Abkürzungen: $\mathrm{LDH}=$ Lactat-Dehydrogenase, $\mathrm{KM}=\mathrm{Kno}$ chenmark. stens ein Teil der Serumenzyme aus dem KM stammen dürfte. Auch die Aktivitäten von Glutamat-Pyruvat- und Glutamat-Oxalacetat-Transaminase, Aldolase, Phosphohexoisomerase und Malat-Dehydrogenase waren im Serum des KM bei unseren Patienten erhöht. RICHrER und KöHLER (4) fanden bei 16 hämatologischen und 36 internistischen Patienten die Erhöhung von Enzymen bei einigen Patienten im Serum des KM. Die Erhöhung war für Lactatdehydrogenase, Malatdehydrogenase und Alkalische Phosphatase verglichen mit den Aktivitäten im venösen Serum statistisch signifikant. Die Erhöhung der Aktivitäten von Enzymen im KM ist nach diesen Autoren durch mikro- oder makroskopische Hämolyse verursacht. ElLIOT und Fleming (6) fanden bei Patienten ohne Anämie und bei Patienten mit megaloblastischer Anämie im Plasma des KM eine höhere Aktivität von $\mathrm{LDH}$ als im Serum. In der Zellfraktion des megaloblastären KM war die LDH-Aktivität höher als im normoblastischen KM. Sie sind deshalb der Meinung, daß die erhöhte Aktivität von Enzymen im venösen Serum aus hohen Enzymkonzentrationen im KM resultiert. Die LDH-Isoenzyme ${ }^{4}$ ) wurden im KM nur wenig untersucht. Pfleiderer und Wachsmuth (7) untersuchten sie im Sektionsmaterial und reihen das KM nach dem Isoenzymmuster in die Gruppe der Organe, die

4) Die schnellste anodische Fraktion nennen wir $\mathrm{LDH}_{1}=\mathrm{H}-\mathrm{Typ}$ von Carn und Mitarb. oder B-Typ von MArkert und Appelie, die kathodische Fraktion $\mathrm{LDH}_{5}=\mathrm{M}-$ Typ oder A-Typ. 